\title{
SIMPLIFIED MODELING OF A THERMO-ACOUSTO-ELECTRIC ENGINE FORCED BY AN EXTERNAL SOUND SOURCE
}

\author{
C. Olivier, G. Poignand*, G. Penelet, and P. Lotton \\ Laboratoire d'Acoustique de l'Université du Maine, UMR CNRS 6613, Avenue Olivier Messiaen, 72085 Le Mans cedex 9 , \\ France. *Corresponding author's e-mail: gaelle.poignand@univ-lemans.fr
}

Keywords: Thermoacoustics, Nonlinear dynamics, Active Control

\section{Introduction}

Thermoacoustic wave-generators are usually designed and optimized using software tools $[1,2]$ based on the linear thermoacoustic theory. These tools enable to predict the operating point of a thermoacoustic engine from the balance between the thermoacoustic amplification process and the numerous nonlinear effects saturating wave amplitude growth, the latter effects being very difficult to describe properly. A non exhaustive list of these effects includes (apart from the thermoacoustic heat pumping accompanying wave amplification) different kinds of streaming, nonlinear acoustic propagation or aerodynamical and thermal entrance effects. These nonlinear effects are responsible for both dissipation of acoustic power and perturbations of the temperature field in the thermoacoustic core, that work together to limit the overall performances of thermal-to-acoustic conversion. Therefore, the development of adequate simulation tools is still needed to describe the high level of complexity of the processes involved above the onset of thermoacoustic instability. Direct numerical simulation seems to be the only way to reproduce quantitatively the effects mentioned above, but it is still limited by large computation times inherent to the complicated physics and multiple time and space scales involved in the description of thermoacoustic engines. Simplified analytical models can help in getting a deeper physical insight about the processes involved, but they are also based on substantial approximations. In this study, we use a simplified modeling of thermoacoustic engines to help understanding recent experimental observations dealing with the external forcing of thermoacoustic oscillations [3-5].

\section{Aims and scope of the study}

Recent proof of concept studies [3,4] performed at LAUM have already shown that the external forcing of thermoacoustic oscillations could be an interesting way to increase the efficiency of a thermoacoustic prime-mover. In particular, we have shown that the adjunction of an adequately tuned electroacoustic feedback loop to a thermo-acousto-electric transducer can lead to the increase of its global efficiency [4]. Using a reference pressure signal from the engine as an input, an auxiliary acoustical source is used to control self-sustained thermoacoustic oscillations in the engine. By tuning the properties of this feedback (in terms of amplification gain and phase-shift relative to the reference signal), the efficiency of the transducer can either be reduced down to the death of the auto-oscillations, or increased, thus generating a greater output power for the same heat input. More recently, we have shown on the same device that the electroacoustic feedback loop is also responsible for the occurrence of hysteretic behavior [5] of both the temperature difference across the regenerator and the efficiency versus the heat input. However, at the present time the multiplicity of the involved nonlinear effects and their complexity does not allow to understand why and how the mechanisms at stake in this process of "active control" enable to increase the global efficiency of the thermoacoustic engine. It is there- 
fore worth considering to make theoretical studies on the topic. This task is however arduous because a pre-requisite is to understand deeply and to describe properly the self-sustained oscillator itself before describing its nonlinear coupling with external sound sources. However, we plan as a first step to find a qualitative description of the dynamics of the system which would be well-suited for the analysis of its bifurcation diagram and its response to external forcing. Such kind of approach is very common in nonlinear dynamics in which complicated systems are sometimes summarized by a few ordinary differential equations.

In this study, a simplified model of a thermo-acousto-electric generator is proposed. This reduced-order model is based on a lumped element approach for the description of the acoustic propagation in the engine, coupled with a resolution of unsteady heat transfer through the thermoacoustic core based on a finite difference scheme. This description allows the study of both transient and steady-state operation of the engine. Applying an external forcing to that model also allows to get a better overview of the effects of the feedback loop on the engine. Notably, by gradually increasing its degree of complexity, it enables to study various parameters, such as the coupling of the auxiliary electrodynamic source with the engine, and the influence of the feedback on both the acoustic field and on the distribution of the temperature field within the thermoacoustic core. This simplified model of a forced thermoacoustic transducer will help to get a better understanding of the phenomena responsible for the complex behaviors induced by the electroacoustic feedback loop on the actual prototype: death of oscillations, efficiency improvement accompanied by a temperature gradient reduction, as well as a hysteretic behavior induced by the electroacoustic feedback loop during the processes of gradual heat input increase followed by a gradual decrease.

\section{References}

[1] Ward W.C., Swift G.W., Design Environement for low Amplitude ThermoAcoustic Engines, J. Acoust. Soc. Am., 95:3671-3672, 1994.

[2] G.W. Swift, Thermoacoustics: A unifying perspective for some engines and refrigerators (Acoustical Society of America, Melville NY, 2001).

[3] C. Desjouy, G. Penelet, P. Lotton, Active control of thermoacoustic amplification in an annular thermoacoustic engine, J. Appl. Phys., 108:114904, 2010.

[4] C. Olivier, G. Penelet, G. Poignand, P. Lotton, "Active control of thermoacoustic amplification in a thermo-acousto-electric engine", J. Appl. Phys., 115:174905, 2014.

[5] G. Poignand, C. Olivier, G. Penelet, P. Lotton, Ân̂Â̆Hysteretic behavior induced by an electroacoustic feedback loop in a thermo-acousto-electric generatorÂă $\hat{A} \dot{z}$, submitted to Appl. Acoust., 2015 